\title{
Application of the Method of Generating Functions to the Derivation of Grad's N-Moment Equations for a Granular Gas
}

\author{
S. H. Noskowicz ${ }^{1 *}$ and D. Serero ${ }^{2}$ \\ ${ }^{1}$ School of Mechanical Engineering, Faculty of engineering, Tel-Aviv University, Ramat-Aviv, \\ Tel-Aviv 69978, Israel \\ ${ }^{2}$ Institute for Multiscale Simulation, Universität Erlangen-Nürnberg, Nägelsbachstraße 49b, \\ 91052 Erlangen, Germany
}

\begin{abstract}
A computer aided method using symbolic computations that enables the calculation of the source terms (Boltzmann) in Grad's method of moments is presented. The method is extremely powerful, easy to program and allows the derivation of balance equations to very high moments (limited only by computer resources). For sake of demonstration the method is applied to a simple case: the one-dimensional stationary granular gas under gravity. The method should find applications in the field of rarefied gases, as well. Questions of convergence, closure are beyond the scope of this article.
\end{abstract}

Key words: Grad's method of moments, granular gas, generating function, computer aided AMS subject classification: 82C40, 82D05

\footnotetext{
${ }^{*}$ Corresponding author. E-mail: henri@eng.tau.ac.il and sh.noskowicz@gmail.com
} 


\section{Introduction}

Professor Isaac Goldhirsch left us in April 2010. The authors of this article are honored and privileged to have been his former Ph.D. students at the Tel-Aviv University, Faculty of Engineering, Department of Fluid Mechanics. This article is dedicated to the memory of Professor Isaac Goldhirsch.

When sufficiently strongly forced a granular system may become fluidized to the point that the grains interact mainly by quasi instantaneous inelastic binary collisions thereby rendering the traditional methods of the kinetic theory of gases applicable to their description [7, 8, 13]. A common approach invokes the Chapman-Enskog expansion properly modified to account for the inelastic nature of granular collisions $[5,9,10,15,27]$ and its extension to moderately dense systems where the two-particle distribution function is represented as the product of one-particle distribution functions, multiplied by the equilibrium pair correlation at contact (Revised Enskog Theory) [12, 14]. The latter consists of a perturbative expansion in the Knudsen number defined as the ratio of the mean free path between collisions (with another particle or with the wall) to a typical length of variation of the macroscopic fields. By nature this expansion is limited to small values of the Knudsen number and high order terms (Burnett, superBurnett) are unstable [4, 26]. High Knudsen number flows are encountered in many important problems including flows at high altitude, shock waves, or flows in micro-scale geometries. The latter type of flows have recently received an increased attention due to the development of micro-electro-mechanical systems (MEMS). In many cases, one has recourse to direct numerical approaches for solving the Boltzmann equation by discretizing the Boltzmann integrals [1], or by a Lagrangian solver which tracks the trajectory of individual particles [2]. Despite some successes this approach is limited by the enormous computational cost involved. Another approach, popularly used to describe micro-devices [17] or in the investigation of shock waves $[22,24,21]$ involves the use of moment methods, and in particular Grad's method of moments [19,3]. Grad's method consists of assuming that the single particle distribution function has the form of a local Maxwellian distribution multiplied by a series of (Hermite) polynomials in the peculiar velocity with time dependent prefactors, which correspond to linear combinations of the moments of the single particle distribution function. In practice the infinite series is truncated at some higher moment which brings along daunting mathematical problems [20, 29, 11]. In this article we shall not concern ourselves with problems of convergence, regularization or stability of the Grad expansion. Instead we begin with the assumption that for any arbitrarily large Knudsen number the Boltzmann equation can be approximately solved by retaining a sufficient number of terms in Grad's expansion. Grad's ansatz serves as a closure for a hierarchy of equations of motions for the moments (or equivalently for the expansion coefficients of the distribution function) that can be derived using the Boltzmann equation. During the last few years, Prof. Isaac Goldhirsch and the present authors (with O. Bar-Lev) have developed a method especially devised for exploiting the capabilities of symbolic processors such as Maple and Mathematica [23, 14]. The goal of the present article is to propose a tool for calculating the Boltzmann source terms emerging in the hierarchy of moment equations to arbitrary order.

This article is organized as follows. Section 2. presents the balance laws for a dilute granular 
gas [18]. The quantity of interest is the source term $\tilde{\chi}$, a tensor whose order increases with the hierarchy to become very rapidly intractable. Section 3. is the core of the article containing the method for calculating $\widetilde{\chi}$. As a way of illustrating the method we derive in section 4 . the equations pertaining to a one-dimensional stationary granular gas (i.e., of infinite transverse extent) under gravity $[6,25]$. We demonstrate the method for the 13 -moment case in the most detailed manner.

\section{Formulation of the problem}

In this article we closely follow Jenkins and Richman [18] to which we refer for a more detailed exposition of the formulation. We consider a gas of inelastic smooth and homogeneous hard spheres of mass $m$, diameter $\sigma$ and coefficient of normal restitution $\alpha$ (or coefficient of inelasticity $\epsilon \equiv 1-\alpha^{2}$ ). The collision between sphere 1 and 2 results in the following velocity transformation:

$$
\begin{aligned}
& \mathbf{c}_{1}^{\prime}=\mathbf{c}_{1}-\frac{1+\alpha}{2}(\mathbf{g} \cdot \mathbf{k}) \mathbf{k} \\
& \mathbf{c}_{2}^{\prime}=\mathbf{c}_{2}+\frac{1+\alpha}{2}(\mathbf{g} \cdot \mathbf{k}) \mathbf{k}
\end{aligned}
$$

where primes denote postcollisional velocities. The relative velocities of spheres 1 and 2 immediately before and after a collision are $\mathbf{g}=\mathbf{c}_{1}-\mathbf{c}_{2}, \mathbf{g}^{\prime}=\mathbf{c}_{1}^{\prime}-\mathbf{c}_{2}^{\prime}$ and $\mathbf{k}$ is a unit vector from the center of sphere 1 to the center of sphere 2 at contact. In what follows in a binary collision between two spheres, primes denote postcollisional quantities.

The single particle distribution and the two-particle distribution are denoted by $f(\mathbf{c}, \mathbf{r}, t)$ and $f^{(2)}\left(\mathbf{c}_{1}, \mathbf{r}_{1}, \mathbf{c}_{2}, \mathbf{r}_{2}, t\right)$, respectively. The mass density is $\rho=n m$, where $n$ is the number density $n \equiv \int f(\mathbf{c}, \mathbf{r}, t) d \mathbf{c}$. The mean value of any particle property $\psi(\mathbf{c})$ is defined as:

$$
\langle\psi\rangle \equiv \frac{1}{n} \int f(\mathbf{c}, \mathbf{r}, t) \psi(\mathbf{c}) d \mathbf{c}
$$

The mean velocity is $\mathbf{u} \equiv\langle\mathbf{c}\rangle$ and the peculiar velocity is $\mathbf{C} \equiv \mathbf{c}-\mathbf{u}$. The temperature is defined by $\left\langle C^{2}\right\rangle \equiv 3 \frac{T}{m}$, the stress tensor is $\mathbf{P} \equiv\langle\rho \mathbf{C C}\rangle$ and the heat flux is $\mathbf{Q} \equiv \frac{1}{2}\left\langle\rho C^{2} \mathbf{C}\right\rangle$. The collisional rate of change of $\psi$ per unit volume at $\mathbf{r}$ is:

$$
\mathcal{C}(\psi)=\sigma^{2} \int_{\mathbf{g} \cdot \mathbf{k}>0}\left(\psi\left(\mathbf{c}_{2}^{\prime}\right)-\psi\left(\mathbf{c}_{2}\right)\right) f^{(2)}\left(\mathbf{c}_{1}, \mathbf{r}_{1}, \mathbf{c}_{2}, \mathbf{r}_{1}+\sigma \mathbf{k}, t\right)(\mathbf{g} \cdot \mathbf{k}) d \mathbf{k} d \mathbf{c}_{1} d \mathbf{c}_{2}
$$

where $\mathbf{r}=\mathbf{r}_{1}+\sigma \mathbf{k}$, which can be written as (see [18]):

$$
\mathcal{C}(\psi)=\chi(\psi)-\frac{\partial}{\partial r_{i}} \Theta_{i}(\psi)-\frac{\partial u_{j}}{\partial r_{i}} \Theta_{i}\left(\frac{\partial \psi}{\partial C_{j}}\right)
$$

where the source term is:

$\chi(\psi)=\frac{\sigma^{2}}{2} \int_{\mathbf{g} \cdot \mathbf{k}>0}\left(\psi\left(\mathbf{c}_{1}^{\prime}\right)+\psi\left(\mathbf{c}_{2}^{\prime}\right)-\psi\left(\mathbf{c}_{1}\right)-\psi\left(\mathbf{c}_{2}\right)\right) f^{(2)}\left(\mathbf{c}_{1}, \mathbf{r}-\sigma \mathbf{k}, \mathbf{c}_{2}, \mathbf{r}, t\right)(\mathbf{g} \cdot \mathbf{k}) d \mathbf{k} d \mathbf{c}_{1} d \mathbf{c}_{2}$ 
and the flux term is:

$$
\begin{array}{r}
\Theta_{i}(\psi)=-\frac{\sigma}{2} \int_{\mathbf{g} \cdot \mathbf{k}>0}\left(\psi\left(\mathbf{c}_{1}^{\prime}\right)-\psi\left(\mathbf{c}_{1}\right)\right) k_{i}\left(1-\frac{\sigma}{2 !} k_{j} \frac{\partial}{\partial r_{j}}+\frac{\sigma^{2}}{3 !} k_{j} k_{m} \frac{\partial^{2}}{\partial r_{j} \partial r_{m}}+\ldots\right) \times \\
f^{(2)}\left(\mathbf{c}_{1}, \mathbf{r}, \mathbf{c}_{2}, \mathbf{r}+\sigma \mathbf{k}, t\right)(\mathbf{g} \cdot \mathbf{k}) d \mathbf{k} d \mathbf{c}_{1} d \mathbf{c}_{2}
\end{array}
$$

The following balance equation holds:

$$
\begin{aligned}
& \frac{D}{D t}\langle\rho \psi\rangle+\langle\rho \psi\rangle \nabla \cdot \mathbf{u}+\frac{\partial}{\partial r_{i}}\left(\left\langle\rho C_{i} \psi\right\rangle+\Theta_{i}(m \psi)\right) \\
& +\rho\left(\frac{D u_{i}}{D t}-\frac{F_{i}}{m}\right)\left\langle\frac{\partial \psi}{\partial C_{i}}\right\rangle+\left(\left\langle\rho C_{i} \frac{\partial \psi}{\partial C_{j}}\right\rangle+\Theta_{i}\left(m \frac{\partial \psi}{\partial C_{j}}\right)\right) \frac{\partial u_{j}}{\partial r_{i}}=\chi(m \psi)
\end{aligned}
$$

where $\mathbf{F}$ is the external force and $\frac{D}{D t} \equiv \frac{\partial}{\partial t}+u_{i} \frac{\partial}{\partial r_{i}}$. Since we consider the dilute limit $\Theta=0$ and:

$$
f^{(2)}\left(\mathbf{c}_{1}, \mathbf{r}-\sigma \mathbf{k}, \mathbf{c}_{2}, \mathbf{r}, t\right)=f\left(\mathbf{c}_{1}, \mathbf{r}, t\right) f\left(\mathbf{c}_{2}, \mathbf{r}, t\right)
$$

The source term then reads:

$$
\chi(\psi)=\frac{\sigma^{2}}{2} \int_{\mathbf{g} \cdot \mathbf{k}>0}\left(\psi\left(\mathbf{c}_{1}^{\prime}\right)+\psi\left(\mathbf{c}_{2}^{\prime}\right)-\psi\left(\mathbf{c}_{1}\right)-\psi\left(\mathbf{c}_{2}\right)\right) f\left(\mathbf{c}_{1}, \mathbf{r}, t\right) f\left(\mathbf{c}_{2}, \mathbf{r}, t\right)(\mathbf{g} \cdot \mathbf{k}) d \mathbf{k} d \mathbf{c}_{1} d \mathbf{c}_{2}
$$

and the balance equation becomes:

$$
\rho \frac{D}{D t}\langle\psi\rangle+\frac{\partial}{\partial r_{i}}\left\langle\rho C_{i} \psi\right\rangle+\rho\left(\frac{D u_{i}}{D t}-\frac{F_{i}}{m}\right)\left\langle\frac{\partial \psi}{\partial C_{i}}\right\rangle+\left\langle\rho C_{i} \frac{\partial \psi}{\partial C_{j}}\right\rangle \frac{\partial u_{j}}{\partial r_{i}}=\chi(m \psi)
$$

Define the N-th moment, source and heat tensors as:

$$
\begin{aligned}
M_{k_{1} \cdots k_{N}} & \equiv\left\langle C_{k_{1}} \cdots C_{k_{N}}\right\rangle \\
\chi_{k_{1} \cdots k_{N}} & \equiv \chi\left(m C_{k_{1}} \cdots C_{k_{N}}\right) \\
Q_{i k_{1} \cdots k_{N}} & \equiv \frac{\rho}{2} M_{i k_{1} \cdots k_{N}}
\end{aligned}
$$

Note that with these definitions $T=\frac{m}{3} M_{i i}, P_{i j}=\rho M_{i j}$ and $Q_{i}=\frac{\rho}{2} M_{i j j}$. Upon substituting $\psi=C_{k_{1}} C_{k_{2}} \ldots C_{k_{N}}(N \geq 4)$ into Eq.(2.4) one obtains the equation of motion for $M_{k_{1} \cdots k_{N}}$ :

$$
\rho \frac{d M_{k_{1} \cdots k_{N}}}{d t}+2 \frac{\partial Q_{i k_{1} \cdots k_{N-1}}}{\partial r_{i}}-N M_{\left(k_{1} \cdots k_{N}\right.} \frac{\partial}{\partial r_{i}} P_{\left.k_{N}\right) i}+2 N \frac{\partial}{\partial r_{i}} Q_{i\left(k_{1} \cdots k_{N-1}\right.} u_{\left.k_{N}\right)}=\chi_{k_{1} \cdots k_{N}}
$$

where parentheses around a set of $\mathrm{N}$ indices denote the sum of all permutations of the indices divided by $N$ ! (see [18] for equations of motion with $N<4$ ). Grad's ansatz for the single-particle distribution function reads [16]:

$$
\begin{aligned}
f(\mathbf{c}, \mathbf{r}, t) & =\left(1-a_{i} \frac{\partial}{\partial c_{i}}+\frac{a_{i j}}{2 !} \frac{\partial^{2}}{\partial c_{i} \partial c_{j}}-\frac{a_{i j k}}{3 !} \frac{\partial^{3}}{\partial c_{i} \partial c_{j} \partial c_{k}}+\cdots\right) f^{0}(\mathbf{c}, \mathbf{r}, t) \\
& =n \frac{m^{\frac{3}{2}}}{(2 \pi T)^{\frac{3}{2}}}\left(1-a_{i} \frac{m C_{i}}{T}+\frac{a_{i j}}{2 !} \frac{m C_{i}}{T} \frac{m C_{j}}{T}-\frac{a_{i j k}}{3 !} \frac{m C_{i}}{T} \frac{m C_{j}}{T} \frac{m C_{k}}{T}+\cdots\right) e^{-\frac{m C^{2}}{2 T}}
\end{aligned}
$$


where $f^{0}$ is the Maxwellian:

$$
f^{0}=n \frac{m^{\frac{3}{2}}}{(2 \pi T)^{\frac{3}{2}}} e^{-\frac{m C^{2}}{2 T}}
$$

It is convenient to define the non-dimensional quantities:

$$
\begin{aligned}
\widetilde{C}_{i} & \equiv \sqrt{\frac{m}{2 T}} C_{i} \\
b_{i_{1} \cdots i_{N}} & \equiv \frac{(-1)^{N}}{N !}\left(\frac{2 m}{T}\right)^{\frac{N}{2}} a_{i_{1} \cdots i_{N}}
\end{aligned}
$$

then Eq.(2.5) becomes:

$$
f(\mathbf{c}, \mathbf{r}, t)=n \frac{m^{\frac{3}{2}}}{(2 \pi T)^{\frac{3}{2}}}\left(1+b_{i} \widetilde{C}_{i}+b_{i j} \widetilde{C}_{i} \widetilde{C}_{j}+b_{i j k} \widetilde{C}_{i} \widetilde{C}_{j} \widetilde{C}_{k}+\cdots\right) e^{-\widetilde{C}^{2}}
$$

It can be shown that the mean value of property $\phi$ is (see [18]):

$$
\langle\phi\rangle=\sum_{p=0}^{\infty} \frac{a_{s_{1} \ldots s_{p}}}{p !}\left\langle\frac{\partial^{p} \phi}{\partial c_{s_{1}} \ldots \partial c_{s_{1}}}\right\rangle^{0}
$$

where $\langle\xi\rangle^{0} \equiv \frac{1}{n} \int \xi(\mathbf{c}) f^{(0)}(\mathbf{c}, \mathbf{r}, t) d \mathbf{c}$. The moment tensors are easily computed:

$$
M_{i_{1} \cdots i_{N}}=N ! \sum_{p=0}^{N} \frac{1}{p !(N-p) !} a_{\left(i_{1} \ldots i_{p}\right.}\left\langle C_{i_{p+1}} \ldots C_{\left.i_{N}\right)}\right\rangle^{0}
$$

However the source term Eq.(2.3) is much more complicated:

$$
\chi_{i_{1} \cdots i_{k}}=\frac{\rho}{2 \pi^{4} \ell}\left(\frac{2 T}{m}\right)^{\frac{k+1}{2}} \sum_{\{\alpha\}} \sum_{\{\beta\}} b_{\alpha_{1} \cdots \alpha_{p}} b_{\beta_{1} \cdots \beta_{q}} \widetilde{\chi}_{i_{1} \cdots i_{k} \alpha_{1} \cdots \alpha_{p} \beta_{1} \cdots \beta_{q}}
$$

where $\ell$ is the mean free path $\ell \equiv \frac{1}{n \pi \sigma^{2}}$ and we encounter a new tensor of size $k+p+q$ (tildes are dropped for the sake of clarity):

$$
\begin{aligned}
& \tilde{\chi}_{i_{1} \cdots i_{k} \alpha_{1} \cdots \alpha_{p} \beta_{1} \cdots \beta_{q}} \equiv \int_{\mathbf{g} \cdot \mathbf{k}>0} d \mathbf{k} d \mathbf{C}_{1} d \mathbf{C}_{2} e^{-\left(C_{1}^{2}+C_{2}^{2}\right)}(\mathbf{g} \cdot \mathbf{k}) \\
& \left(C_{1 i_{1}}^{\prime} \cdots C_{1 i_{k}}^{\prime}+C_{2 i_{1}}^{\prime} \cdots C_{2 i_{k}}^{\prime}-C_{1 i_{1}} \cdots C_{1 i_{k}}-C_{2 i_{1}} \cdots C_{2 i_{k}}\right) C_{1 \alpha_{1}} \cdots C_{1 \alpha_{p}} C_{2 \beta_{1}} \cdots C_{2 \beta_{q}}
\end{aligned}
$$

This tensor $\tilde{\chi}$ becomes forbiddingly tedious to calculate as its order increases. As mentioned, the main goal of the present article to present a computer-aided method allowing the automatic calculation of this tensor to any order. 


\section{Calculation of the tensor $\tilde{\chi}$}

The tensor $\widetilde{\chi}_{i_{1} \cdots i_{k} \alpha_{1} \cdots \alpha_{p} \beta_{1} \cdots \beta_{q}}$ is not velocity dependent, i.e. it is a constant tensor. It follows from tensorial considerations that it is a linear combination of products of Kronecker's delta's of the indices (and vanishes for odd values of $k+p+q)$. There are $(k+p+q-1)$ !! such terms. For example the sixth order tensor (15 terms) $\tilde{\chi}_{i_{1} i_{2} \alpha_{1} \alpha_{2} \beta_{1} \beta_{2}}$ is of the form:

$$
\tilde{\chi}_{i_{1} i_{2} \alpha_{1} \alpha_{2} \beta_{1} \beta_{2}}=A_{1} \delta_{i_{1}, i_{2}} \delta_{\alpha_{1}, \beta_{1}} \delta_{\alpha_{2}, \beta_{2}}+A_{2} \delta_{\alpha_{1}, \alpha_{2}} \delta_{\beta_{2}, \beta_{1}} \delta_{i_{1}, i_{2}}+\cdots+A_{15} \delta_{\beta_{2}, \beta_{1}} \delta_{\alpha_{2}, i_{2}} \delta_{i_{1}, \alpha_{1}}
$$

It is convenient to define a vector of pair-partitions $\Pi$ whose entries correspond to specific partitions. In the example: $\Pi(1)=\left\{\left(i_{1}, i_{2}\right)\left(\alpha_{1}, \beta_{1}\right)\left(\alpha_{2}, \beta_{2}\right)\right\}, \ldots, \Pi(15)=\left\{\left(\beta_{2}, \beta_{1}\right)\left(\alpha_{2}, i_{2}\right)\left(i_{1}, \alpha_{1}\right)\right\}$ The corresponding product of deltas is defined as $\delta_{\Pi(i)}$. There are exactly as many constant $A_{i}$ 's as the number of pair-partitions $N_{\text {Part }}$. Hence:

$$
\tilde{\chi}_{i_{1} \cdots i_{k} \alpha_{1} \cdots \alpha_{p} \beta_{1} \cdots \beta_{q}}=\sum_{i=1}^{N_{\text {Part }}} A_{i} \delta_{\Pi(i)}
$$

Upon multiplying and contracting $\tilde{\chi}$ by all the $\delta_{\Pi(i)}$ one obtains $N_{\text {Part }}$ equations for the $\left\{A_{i}\right\}$. In the example: $\delta_{\Pi(1)}=\delta_{i_{1}, i_{2}} \delta_{\alpha_{1}, \beta_{1}} \delta_{\alpha_{2}, \beta_{2}}, \delta_{\Pi(2)}=\delta_{i_{1}, i_{2}} \delta_{\alpha_{1}, \alpha_{2}} \delta_{\beta_{1}, \beta_{2}}, \ldots$ so that:

$$
\begin{aligned}
& \delta_{\Pi(1)} \widetilde{\chi}_{i_{1} i_{2} \alpha_{1} \alpha_{2} \beta_{1} \beta_{2}}=27 A_{1}+9 A_{2}+\cdots+3 A_{15} \\
& \delta_{\Pi(2)} \widetilde{\chi}_{i_{1} i_{2} \alpha_{1} \alpha_{2} \beta_{1} \beta_{2}}=9 A_{1}+27 A_{2}+\cdots+9 A_{1} 5
\end{aligned}
$$

Applying the $\delta_{\Pi(i)}$ operation under the integral sign defining $\tilde{\chi}$ in Eq.(2.6) in the left hand side of the above system yields a scalar function of the velocities. In the example:

$$
\begin{aligned}
& \delta_{\Pi(15)} \widetilde{\chi}_{i_{1} 1_{2} \alpha_{1} \alpha_{2} \beta_{1} \beta_{2}}=\int_{\mathbf{g} \cdot \mathbf{k}>0} d \mathbf{k} d \mathbf{C}_{1} d \mathbf{C}_{2} e^{-\left(C_{1}^{2}+C_{2}^{2}\right)}(\mathbf{g} \cdot \mathbf{k}) \times \\
& \delta_{\beta_{2}, \beta_{1}} \delta_{\alpha_{2}, i_{2}} \delta_{i_{1}, \alpha_{1}}\left(C_{1 i_{1}}^{\prime} C_{1 i_{2}}^{\prime}+C_{2 i_{1}}^{\prime} C_{2 i_{2}}^{\prime}-C_{1 i_{1}} C_{1 i_{2}}-C_{2 i_{1}} C_{2 i_{2}}\right) C_{1 \alpha_{1}} C_{1 \alpha_{2}} C_{2 \beta_{1}} C_{2 \beta_{2}} \\
= & \int_{\mathbf{g} \cdot \mathbf{k}>0} d \mathbf{k} d \mathbf{C}_{1} d \mathbf{C}_{2} e^{-\left(C_{1}^{2}+C_{2}^{2}\right)}(\mathbf{g} \cdot \mathbf{k})\left(\left(\mathbf{C}_{1}^{\prime} \cdot \mathbf{C}_{1}\right)^{2}+\left(\mathbf{C}_{2}^{\prime} \cdot \mathbf{C}_{1}\right)^{2}-C_{1}^{4}-\left(\mathbf{C}_{1} \cdot \mathbf{C}_{2}\right)^{2}\right) C_{2}^{2}
\end{aligned}
$$

Integrals of the type of Eq.(3.2) can obviously be calculated by hand but the integrals become rapidly overwhelming at higher orders. To simplify the labor we have invented a method based on a single generating function, the supergenerating function defined as follows:

$$
I_{1}=\int_{\mathbf{g} \cdot \mathbf{k}>0}(\mathbf{g} \cdot \mathbf{k}) e^{-F} d \mathbf{k} d \mathbf{C}_{1} d \mathbf{C}_{2}
$$

where:

$$
F=b_{1} C_{1}^{2}+b_{2} C_{2}^{2}+b_{3} C_{1}^{\prime 2}+b_{4} C_{2}^{\prime 2}+\frac{x}{2}\left(\mathbf{C}_{1}^{\prime}+\mathbf{C}_{1}\right)^{2}+\frac{y}{2}\left(\mathbf{C}_{1}^{\prime}+\mathbf{C}_{2}\right)^{2}+\frac{z}{2}\left(\mathbf{C}_{1}^{\prime}+\mathbf{C}_{2}^{\prime}\right)^{2}
$$


The calculation of $I_{1}\left(2 \mathrm{D}\right.$ and 3D) is presented in Appendix A. By taking derivatives of $I_{1}$ with respect to $b_{i}, x, y$ and $z$, one can straightforwardly obtain integrals of the type of Eq.(3.2) (see Appendix A). Returning to the example we have:

$$
\begin{aligned}
& \delta_{\Pi(15) \tilde{\chi}_{i_{1} \alpha_{1} \alpha_{2} \beta_{1} \beta_{2} \beta_{3}}=} \\
& {\left[\left(-\frac{\partial}{\partial x}+\frac{1}{2} \frac{\partial}{\partial b_{3}}+\frac{1}{2} \frac{\partial}{\partial b_{1}}\right)^{2}+\left(\frac{\partial}{\partial x}-\frac{\partial}{\partial z}-\frac{\partial}{\partial b_{1}}+\frac{1}{2} \frac{\partial}{\partial b_{2}}-\frac{1}{2} \frac{\partial}{\partial b_{3}}\right)^{2}\right.} \\
& \left.-\left(-\frac{\partial}{\partial b_{1}}\right)^{2}-\left(-\frac{\partial}{\partial z}+\frac{1}{2} \frac{\partial}{\partial b_{1}}+\frac{1}{2} \frac{\partial}{\partial b_{2}}\right)^{2}\right]\left(-\frac{\partial}{\partial b_{1}}\right) I_{1} \\
= & \left(-2 \frac{\partial^{3}}{\partial b_{1} \partial x^{2}}+2 \frac{\partial^{3}}{\partial b_{1} \partial x \partial b_{3}}+3 \frac{\partial^{3}}{\partial b_{1}^{2} \partial x}-\frac{1}{2} \frac{\partial^{3}}{\partial b_{1} \partial b_{3}^{2}}-\frac{3}{2} \frac{\partial^{3}}{\partial b_{1}^{2} \partial b_{3}}+2 \frac{\partial^{3}}{\partial b_{1} \partial x \partial z}\right. \\
+ & \left.-\frac{\partial^{3}}{\partial b_{1} \partial x \partial b_{2}}-3 \frac{\partial^{3}}{\partial b_{1}^{2} \partial z \partial b_{2}}-\frac{\partial^{3}}{\partial b_{1} \partial z \partial b_{3}}+\frac{3}{2} \frac{\partial^{3}}{\partial b_{1}^{2} \partial b_{2}}+\frac{1}{2} \frac{\partial^{3}}{\partial b_{1} \partial b_{2} \partial b_{3}}\right) I_{1}
\end{aligned}
$$

This expression must be computed for $b_{1}=b_{2}=1$ and all other variables equal to zero. These quantities can be conveniently calculated with the help of symbolic processor such as Maple or Mathematica. The linear system for the coefficients $A_{i}$ is completely determined and can be solved.

\section{Stationary one-dimensional granular gas under gravity}

To illustrate the method we consider a simple system: a stationary one-dimensional granular gas under gravity. Energy is continuously provided from below $(z=0)$. The system is infinite and homogeneous in the transverse dimensions $(x, y)$. We content ourselves with the derivation of the equations and will relegate the study of the properties of the resulting differential system to a forthcoming publication. We shall ignore the difficult problem of defining appropriate boundary conditions on the bottom (e.g., Maxwell boundary conditions). Since $\mathbf{u}=0$, Eq.(2.4) becomes:

$$
\frac{\partial}{\partial z}\left\langle\rho C_{z} \psi\right\rangle+g \rho\left\langle\frac{\partial \psi}{\partial C_{z}}\right\rangle=\chi(\psi)
$$

Since the system depends on $\left(C_{z}, C^{2}\right)$, it is convenient to use the shorthand notation:

$$
\begin{aligned}
M_{N, p} & \equiv\left\langle C^{2 N} C_{z}^{p}\right\rangle \\
\chi_{N, p} & \equiv \chi\left(m C^{2 N} C_{z}^{p}\right)=\frac{\rho}{2 \pi^{4} \ell}\left(\frac{2 T}{m}\right)^{N+\frac{p+1}{2}} \widetilde{\chi}_{N, p}
\end{aligned}
$$

Note that:

$$
\begin{aligned}
& M_{1,0}=\frac{3 T}{m} \\
& M_{0,1}=0 \\
& M_{0,0}=1
\end{aligned}
$$


In terms of $M_{N, p}$ and $\chi_{N, p}$ Eq.(4.1) reads:

$$
\frac{d}{d z}\left(\rho M_{N, p+1}\right)+g \rho\left(2 N M_{N-1, p+1}+p M_{N, p-1}\right)=\chi_{N, p}
$$

For future reference, $N=0, p=0$ yields the trivial equality: $0=0$ and for $N=0, p=1$ :

$$
\frac{d M_{0,2}}{d z}=-\rho g
$$

In the 1D case, Eq.(2.5) can be written in the form:

$$
f=f^{0} \sum_{\alpha=0}^{\infty} \sum_{q=0}^{\infty} \frac{2^{\frac{q}{2}}}{q !} \mu^{(q, \alpha)} \widetilde{C}^{2 \alpha} \widetilde{C}_{z}^{q}
$$

thereby defining the coefficients $\mu^{(q, \alpha)}$. In terms of these coefficients the moments read:

$$
M_{N, p}=\frac{2}{\pi^{\frac{3}{2}}}\left(\frac{2 T}{m}\right)^{\frac{p}{2}+N} \sum_{\alpha=0}^{\infty} \sum_{q=0}^{\infty} \frac{2^{\frac{q}{2}}}{q !} \mu^{(q, \alpha)} \delta_{p+q, \text { even }} A_{\alpha+N, p+q} \equiv\left(\frac{2 T}{m}\right)^{\frac{p}{2}+N} \widetilde{M}_{N, p}
$$

defining the non-dimensionalized $\widetilde{M}_{N, p}$ and:

$$
\begin{aligned}
A_{\ell k} & \equiv \int \Theta\left(C_{z}\right) e^{-C^{2}} C^{2 \ell} C_{z}^{k} d \mathbf{C}=2 \pi \int_{0}^{\infty} \Theta\left(C_{z}\right) e^{-C^{2}} C^{2\left(\ell+\frac{k}{2}+1\right)} d C \int_{0}^{\frac{\pi}{2}} \sin \theta \cos ^{k} \theta d \theta \\
& =\frac{2 \pi}{k+1} \int_{0}^{\infty} e^{-C^{2}} C^{2\left(\ell+\frac{k}{2}+1\right)} d C=\frac{\pi}{k+1} \Gamma\left(\ell+\frac{k+3}{2}\right)
\end{aligned}
$$

Eqs.(4.4) become:

$$
\begin{aligned}
& \widetilde{M}_{1,0}=\frac{3}{2} \\
& \widetilde{M}_{0,0}=1 \\
& \widetilde{M}_{0,1}=0
\end{aligned}
$$

It is convenient to measure heights in units of mean free path:

$$
\xi=\int_{0}^{z} \frac{d z^{\prime}}{\ell\left(z^{\prime}\right)}
$$

and to define the non-dimensionalized densities and temperatures $\widetilde{n} \equiv \frac{n}{n(0)}$ and $\widetilde{T} \equiv \frac{T}{T(0)}$ where $n(0)$ and $T(0)$ are the density and temperature of the gas at the bottom. Eq.(4.5) becomes

$$
\begin{aligned}
\widetilde{M}_{N, p+1} \frac{d \log \widetilde{n}}{d \xi} & +\left(\frac{p+1}{2}+N\right) \widetilde{M}_{N, p+1} \frac{d \log \widetilde{T}}{d \xi}+\frac{d}{d \xi} \widetilde{M}_{N, p+1} \\
& =\frac{\widetilde{\chi}_{N p}}{2 \pi^{4}}-\frac{K n}{\widetilde{n} \widetilde{T}}\left(N \widetilde{M}_{N-1, p+1}+\frac{p}{2} \widetilde{M}_{N, p-1}\right)
\end{aligned}
$$


where we have defined a Knudsen number $K n$ by:

$$
K n=\frac{m g \ell(0)}{T(0)}
$$

The linear infinite first-order differential system Eqs.(4.12) for the variables $\tilde{n}, \tilde{T}$ and the set $\mu^{(n, \alpha)}$ is to be solved with the initial conditions: $\tilde{n}(0)=\tilde{T}(0)=1$ and $\mu^{(n, \alpha)}(0)$. In order to calculate $\mu^{(n, \alpha)}(0)$ one needs boundary conditions at the bottom. A common choice is the Maxwell boundary conditions (see Appendix B). Notice that by integrating Eq.(4.6) one obtains a relation between $\widetilde{M}_{0,2}$, the total number of particles per unit area, $N_{T}$, and $K n$.

$$
\widetilde{M}_{0,2}(0)=\frac{m g N_{T}}{n(0) T(0)}=\frac{1}{2} N_{T}\left(\pi \sigma^{2}\right) K n
$$

Similarly, the heat flux at the bottom is related to $\widetilde{M}_{1,1}$ by:

$$
Q_{z}(0)=\frac{m}{2} n(0)\left(\frac{2 T(0)}{m}\right)^{\frac{3}{2}} \widetilde{M}_{1,1}(0)
$$

Notice that the matrix corresponding to the 1.h.s. of Eqs.(4.12) may not be of full rank (as e.g., in the 13 moment case, see below). After reducing this matrix to row echelon form, the augmented matrix assumes the form

$$
\left(\begin{array}{ccccc}
1 & * & * & \ldots & * \\
0 & 1 & * & \ldots & * \\
0 & 0 & 1 & \ldots & * \\
\vdots & \vdots & \vdots & \vdots & \vdots \\
0 & 0 & 0 & 0 \ldots 0 & R H S_{1} \\
0 & 0 & 0 & 0 \ldots 0 & R H S_{2} \\
\vdots & \vdots & \vdots & \vdots & \vdots
\end{array}\right)
$$

where $R H S_{1}, R H S_{2}, \ldots$ are the right hand sides obtained after augmenting the matrix of the system. The system is soluble if $R H S_{1}=0, R H S_{2}=0, \ldots$ These algebraic equations will supplement the system. By taking the derivative of the constraints with respect to $\xi$, one obtains again a full-rank system. The constraints must be satisfied on the boundary: $R H S_{1}(0)=0$, $R H S_{2}(0)=\cdots=0$. In the following we first show how the calculation of $\chi_{N, p}$ simplifies in the one-dimensional case. Finally we calculate the 13-moments case in detail for definiteness.

\subsection{Calculation of $\widetilde{\chi}_{N, p}$}

Since by definition $\chi_{N, p} \equiv \chi\left(m C^{2 N} C_{z}^{p}\right)$ one has:

$$
\widetilde{\chi}_{N, p}=\sum_{\alpha_{1}=0}^{\infty} \sum_{\alpha_{2}=0}^{\infty} \sum_{q_{1}=0}^{\infty} \sum_{q_{2}=0}^{\infty} \frac{1}{q_{1} !} \frac{1}{q_{2} !} \mu^{\left(q_{1}, \alpha_{1}\right)} \mu^{\left(q_{2}, \alpha_{2}\right)} 2^{\frac{q_{1}+q_{2}}{2}} F\left(N, p, q_{1}, q_{2}, \alpha_{1}, \alpha_{2}\right)
$$


where:

$$
\begin{aligned}
F\left(N, p, q_{1}, q_{2}, \alpha_{1}, \alpha_{2}\right)= & \int_{(\mathbf{g} \cdot \mathbf{k})>0} e^{-\left(C_{1}^{2}+C_{2}^{2}\right)}(\mathbf{g} \cdot \mathbf{k}) d \mathbf{k} d C_{1} d C_{2} \times \\
& \left(C_{1}^{\prime 2 N} C_{1 z}^{\prime p}+C_{2}^{\prime 2 N} C_{2 z}^{\prime p}-C_{1}^{2 N} C_{1 z}^{p}-C_{2}^{2 N} C_{2 z}^{p}\right) C_{1}^{2 \alpha_{1}} C_{1 z}^{q_{1}} C_{2}^{2 \alpha_{2}} C_{2 z}^{q_{2}}
\end{aligned}
$$

Next, we define the generalized tensor $\tilde{\chi}$ :

$$
\begin{aligned}
& \tilde{\chi}_{i_{1} \cdots i_{p} j_{1} \cdots j_{q_{1}} \ell_{1} \cdots \ell_{q_{2}}}^{\left(N, \alpha_{1}, \alpha_{2}\right)} \equiv \int d \mathbf{k} d \mathbf{C}_{1} d \mathbf{C}_{2} e^{-\left(C_{1}^{2}+C_{2}^{2}\right)}(\mathbf{g} \cdot \mathbf{k}) C_{1}^{2 \alpha_{1}} C_{2}^{2 \alpha_{2}} \times \\
& \left(C_{1}^{\prime 2 N} C_{1 i_{1} \ldots}^{\prime} C_{1 i_{p}}^{\prime}+C_{2}^{\prime 2 N} C_{2 i_{1} \cdots}^{\prime} C_{2 i_{p}}^{\prime}-C_{1}^{2 N} C_{1 i_{1} \cdots} C_{1 i_{p}}-C_{2}^{2 N} C_{2 i_{1} \cdots C_{2 i_{p}}}\right) C_{1_{j_{1}}} \cdots C_{1_{j_{q_{1}}}} C_{2_{\ell_{1}}} \cdots C_{2_{\ell_{q_{2}}}}
\end{aligned}
$$

One has:

$$
\widetilde{\chi}_{i_{1} \cdots i_{p} j_{1} \cdots j_{q_{1}} \ell_{1} \cdots \ell_{q_{2}}}^{\left(N, \alpha_{1}, \alpha_{2}\right)}=\sum_{i=1}^{N_{\text {Part }}} A_{i}^{\left(N, \alpha_{1}, \alpha_{2}\right)} \delta_{\Pi(i)}
$$

where the sum is over all pair-partitions of the set of indices: $\left\{i_{1}, \cdots, i_{p}, j_{1}, \cdots, j_{q_{1}}, \ell_{1}, \cdots, \ell_{q_{2}}\right\}$. Then:

$$
F\left(N, p, q_{1}, q_{2}, \alpha_{1}, \alpha_{2}\right)=\tilde{\chi}_{z \cdots z z \cdots z z \cdots}^{\left(N, \alpha_{1}, \alpha_{2}\right)}
$$

Here all deltas are equal to 1 so that:

$$
F\left(N, p, q_{1}, q_{2}, \alpha_{1}, \alpha_{2}\right)=\sum_{i=1}^{N_{\text {Part }}} A_{i}^{\left(N, \alpha_{1}, \alpha_{2}\right)}
$$

To obtain the sum on the r.h.s. of the latter equation, one can proceed as follows. Multiply both sides of Eq.(4.16) by $\sum_{i^{\prime}=1}^{N_{\text {Part }}} \delta_{\Pi\left(i^{\prime}\right)}$. Since:

$$
\sum_{i^{\prime}=1}^{N_{\text {Part }}} \sum_{i=1}^{N_{\text {Part }}} \delta_{\Pi\left(i^{\prime}\right)} \delta_{\Pi(i)}=\mathcal{N}\left(p+q_{1}+q_{2}\right)
$$

where $\mathcal{N}\left(p+q_{1}+q_{2}\right)$ is a number depending on the number of indices, one obtains:

$$
F\left(N, p, q_{1}, q_{2}, \alpha_{1}, \alpha_{2}\right)=\sum_{i=1}^{N_{\text {Part }}} A_{i}^{\left(N, \alpha_{1}, \alpha_{2}\right)}=\frac{1}{\mathcal{N}\left(p+q_{1}+q_{2}\right)} \sum_{i=1}^{N_{\text {Part }}} \widetilde{\chi}_{i_{1} \cdots i_{p} j_{1} \cdots j_{q_{1}} \ell_{1} \cdots \ell_{q_{2}}}^{\left(N, \alpha_{1}, \alpha_{2}\right)} \delta_{\Pi(i)}
$$


For example:

$$
\begin{aligned}
& F\left(N, 1,1,2, \alpha_{1}, \alpha_{2}\right)=\frac{1}{3} \times \tilde{\chi}_{i j \ell_{1} \ell_{2}}^{\left(N, \alpha_{1}, \alpha_{2}\right)}\left\{\delta_{i, j} \delta_{\ell_{1}, \ell_{2}}+\delta_{i, \ell_{1}} \delta_{j, \ell_{2}}+\delta_{i, \ell_{2}} \delta_{j, \ell_{1}}\right\} \\
= & \frac{1}{3} \int d \mathbf{k} d \mathbf{C}_{1} d \mathbf{C}_{2} e^{-\left(C_{1}^{2}+C_{2}^{2}\right)}(\mathbf{g} \cdot \mathbf{k}) C_{1}^{2 \alpha_{1}} C_{2}^{2 \alpha_{2}} \times \\
& \frac{1}{3}\left(C_{1}^{\prime 2 N} C_{1_{i}}^{\prime}+C_{2}^{\prime 2 N} C_{2_{i}}^{\prime}-C_{1}^{2 N} C_{1_{i}}-C_{2}^{2 N} C_{2_{i}}\right) C_{1_{j}} C_{\ell_{\ell_{1}}} C_{2_{\ell_{2}}}\left\{\delta_{i, j} \delta_{\ell_{1}, \ell_{2}}+\delta_{i, \ell_{1}} \delta_{j, \ell_{2}}+\delta_{i, \ell_{2}} \delta_{j, \ell_{1}}\right\} \\
= & \frac{1}{3} \int d \mathbf{k} d \mathbf{C}_{1} d \mathbf{C}_{2} e^{-\left(C_{1}^{2}+C_{2}^{2}\right)}(\mathbf{g} \cdot \mathbf{k}) C_{1}^{2 \alpha_{1}} C_{2}^{2 \alpha_{2}} \times \\
& \left(C_{1}^{\prime 2 N} C_{1_{i}}^{\prime}+C_{2}^{\prime 2 N} C_{2_{i}}^{\prime}-C_{1}^{2 N} C_{1_{i}}-C_{2}^{2 N} C_{2_{i}}\right)\left(C_{1_{i}} C_{2}^{2}+\left(C_{1_{i}}+C_{2_{i}}\right)\left(\mathbf{C}_{1} \cdot \mathbf{C}_{2}\right)\right) \\
= & \frac{1}{3} \int d \mathbf{k} d \mathbf{C}_{1} d \mathbf{C}_{2} e^{-\left(C_{1}^{2}+C_{2}^{2}\right)}(\mathbf{g} \cdot \mathbf{k}) C_{1}^{2 \alpha_{1}} C_{2}^{2 \alpha_{2}} \times \\
& \left\{C_{1}^{\prime 2 N}\left[\left(\mathbf{C}_{1} \cdot \mathbf{C}_{1}^{\prime}\right) C_{2}^{2}+\left(\mathbf{C}_{1} \cdot \mathbf{C}_{1}^{\prime}\right)\left(\mathbf{C}_{1} \cdot \mathbf{C}_{2}\right)+\left(\mathbf{C}_{1}^{\prime} \cdot \mathbf{C}_{2}\right)\left(\mathbf{C}_{1} \cdot \mathbf{C}_{2}\right)\right]\right. \\
& +C_{2}^{2 N}\left[\left(\mathbf{C}_{1} \cdot \mathbf{C}_{2}^{\prime}\right) C_{2}^{2}+\left(\mathbf{C}_{1} \cdot \mathbf{C}_{2}^{\prime}\right)\left(\mathbf{C}_{1} \cdot \mathbf{C}_{2}\right)+\left(\mathbf{C}_{2}^{\prime} \cdot \mathbf{C}_{2}\right)\left(\mathbf{C}_{1} \cdot \mathbf{C}_{2}\right)\right] \\
& -C_{1}^{2 N}\left[C_{1}^{2} C_{2}^{2}+\left(\mathbf{C}_{1} \cdot \mathbf{C}_{2}^{\prime}\right)^{2}+\left(\mathbf{C}_{1} \cdot \mathbf{C}_{2}\right) C_{1}^{2}\right] \\
& \left.-C_{2}^{2 N}\left[\left(\mathbf{C}_{1} \cdot \mathbf{C}_{2}\right) C_{2}^{2}+\left(\mathbf{C}_{1} \cdot \mathbf{C}_{2}\right)^{2}+\left(\mathbf{C}_{1} \cdot \mathbf{C}_{2}\right) C_{2}^{2}\right]\right\}
\end{aligned}
$$

We consider next the 13 moments case.

\subsection{Grad's 13 moments}

The 13 variables of the system are $\rho, u_{i}, \mathrm{~T}, a_{i j}$ (symmetric and traceless) and $a_{i j j}$ and:

$$
f=f^{0}\left(1+\frac{1}{2}\left(\frac{m}{T}\right)^{2} a_{i j} C_{i} C_{j}+\frac{1}{10}\left(\frac{m}{T}\right)^{2} a_{i j j}\left(\left(\frac{m}{T}\right) C^{2}-5\right) C_{i}\right)
$$

For a one-dimensional system $a_{x x}=a_{y y}=-\frac{a_{z z}}{2}$ and Eq.(4.17) simplifies:

$$
\begin{aligned}
f & =f^{0}\left(1+\frac{a_{z z}}{2}\left(\frac{m}{T}\right)^{2}\left(-\frac{1}{2}\left(C^{2}-C_{z}^{2}\right)+C_{z}^{2}\right)+\frac{a_{z j j}}{10}\left(\frac{m}{T}\right)^{2}\left(\left(\frac{m}{T}\right) C^{2}-5\right) C_{z}\right) \\
& =\frac{n m^{\frac{3}{2}}}{(2 \pi T)^{\frac{3}{2}}} e^{-\widetilde{C}^{2}}\left(1+\frac{a_{z z}}{2}\left(\frac{m}{T}\right)\left(3 \widetilde{C}_{z}^{2}-\widetilde{C}^{2}\right)+\frac{2 a_{z j j}}{5}\left(\frac{m}{2 T}\right)^{\frac{3}{2}}\left(2 \widetilde{C}^{2}-5\right) \widetilde{C}_{z}\right)
\end{aligned}
$$


The sum in Eq.(4.7) is truncated at $q \leq N_{m}$ and $\alpha \leq N_{s}$. It is easy to see that Eq.(4.17) is recovered if $N_{m}=2$ and $N_{s}=1$. Comparing to Eq.(4.18):

$$
\begin{aligned}
\mu^{(0,0)} & =1 \\
\mu^{(2,0)} & =\frac{3}{2}\left(\frac{m}{T}\right) a_{z z}=\frac{3}{2} A \\
\mu^{(1,1)} & =\frac{1}{5}\left(\frac{m}{T}\right)^{\frac{3}{2}} a_{z j j}=\frac{2 \sqrt{2}}{5} B \\
\mu^{(1,0)} & =-\frac{1}{2}\left(\frac{m}{T}\right)^{\frac{3}{2}} a_{z j j}=-\sqrt{2} B \\
\mu^{(0,1)} & =-\frac{1}{2}\left(\frac{m}{T}\right) a_{z z}=-\frac{1}{2} A \\
\mu^{(2,1)} & =0
\end{aligned}
$$

where:

$$
A \equiv \frac{m}{T} a_{z z} ; \quad B \equiv\left(\frac{m}{2 T}\right)^{\frac{3}{2}} a_{z j j}
$$

In terms of the new parameters, $A$ and $B$, the moments read:

$$
\widetilde{M}_{N, p}=\frac{2}{\pi^{\frac{3}{2}}} A_{N, p} \delta_{p, \text { even }}+\frac{A}{\pi^{\frac{3}{2}}}\left(3 A_{N, p+2}-A_{N+1, p}\right) \delta_{p, \text { even }}+\frac{2 B}{\pi^{\frac{3}{2}}}\left(\frac{4}{5} A_{N+1, p+1}-2 A_{N, p+1}\right) \delta_{p, \text { odd }}
$$

It is readily verified that $\widetilde{M}_{0,0}=1, \widetilde{M}_{0,1}=0, \widetilde{M}_{1,0}=\frac{3}{2}$ and:

$$
\begin{aligned}
\widetilde{M}_{1,1} & =B \\
\widetilde{M}_{0,2} & =\frac{1+A}{2} \\
\widetilde{M}_{0,3} & =\frac{3}{5} B \\
\widetilde{M}_{1,2} & =\frac{5+7 A}{4}
\end{aligned}
$$

The variables are: $\widetilde{n}, \widetilde{T}, A$ and $B$.

The first four equations of the hierarchy: $((N, p)=(0,1),(0,2),(1,0),(1,1))$ yield the system:

$$
\begin{aligned}
(1+A) \frac{d \log \tilde{n}}{d \xi}+(1+A) \frac{d \log \widetilde{T}}{d \xi}+\frac{d A}{d \xi} & =-\frac{K n}{\widetilde{n} \widetilde{T}} \\
B \frac{d \log \widetilde{n}}{d \xi}+\frac{3}{2} B \frac{d \log \widetilde{T}}{d \xi}+\frac{d B}{d \xi} & =\frac{1}{2 \pi^{4}} \widetilde{\chi}_{1,0} \\
B \frac{d \log \tilde{n}}{d \xi}+\frac{3}{2} B \frac{d \log \widetilde{T}}{d \xi}+\frac{d B}{d \xi} & =\frac{5}{3} \frac{1}{2 \pi^{4}} \widetilde{\chi}_{0,2} \\
(5+7 A) \frac{d \log \widetilde{n}}{d \xi}+2(5+7 A) \frac{d \log \widetilde{T}}{d \xi}+7 \frac{d A}{d \xi} & =\frac{2}{\pi^{4}} \widetilde{\chi}_{1,1}-\frac{K n}{\widetilde{n} \widetilde{T}}(5+2 A)
\end{aligned}
$$


The second and third rows of the 1.h.s. of the system are equal so that a row should be replaced by the constraint $\frac{5}{3} \widetilde{\chi}_{0,2}=\widetilde{\chi}_{1,0}$. The final system is thus a differential system plus a constraint:

$$
\begin{aligned}
(1+A) \frac{d \log \tilde{n}}{d \xi}+(1+A) \frac{d \log \widetilde{T}}{d \xi}+\frac{d A}{d \xi} & =-\frac{K n}{\widetilde{n} \widetilde{T}} \\
B \frac{d \log \widetilde{n}}{d \xi}+\frac{3}{2} B \frac{d \log \widetilde{T}}{d \xi}+\frac{d B}{d \xi} & =\frac{1}{2 \pi^{4}} \widetilde{\chi}_{1,0} \\
(5+7 A) \frac{d \log \widetilde{n}}{d \xi}+2(5+7 A) \frac{d \log \widetilde{T}}{d \xi}+7 \frac{d A}{d \xi} & =\frac{2}{\pi^{4}} \widetilde{\chi}_{1,1}-\frac{K n}{\widetilde{n} \widetilde{T}}(5+2 A) \\
\frac{5}{3} \widetilde{\chi}_{0,2}=\widetilde{\chi}_{1,0} &
\end{aligned}
$$

There remains to calculate the terms on the r.h.s: $\widetilde{\chi}_{0,2}, \widetilde{\chi}_{1,0}$ and $\widetilde{\chi}_{1,1}$.

Eq.(4.15) is:

$$
\begin{aligned}
\tilde{\chi}_{N, p} & =\left(\mu^{(0,0)}\right)^{2} F(N, p, 0,0,0,0)+\left(\mu^{(0,1)}\right)^{2} F(N, p, 0,0,1,1) \\
& +2\left(\mu^{(1,0)}\right)^{2} F(N, p, 1,1,0,0)+2\left(\mu^{(1,1)}\right)^{2} F(N, p, 1,1,1,1) \\
& +\left(\mu^{(2,0)}\right)^{2} F(N, p, 2,2,0,0)+2 \mu^{(0,0)} \mu^{(0,1)} F(N, p, 0,0,0,1) \\
& +2^{\frac{3}{2}} \mu^{(0,0)} \mu^{(1,0)} F(N, p, 0,1,0,0)+2^{\frac{3}{2}} \mu^{(0,0)} \mu^{(1,1)} F(N, p, 0,1,0,1) \\
& +2 \mu^{(0,0)} \mu^{(2,0)} F(N, p, 0,2,0,0)+2^{\frac{3}{2}} \mu^{(0,1)} \mu^{(1,0)} F(N, p, 0,1,1,0) \\
& +2^{\frac{3}{2}} \mu^{(0,1)} \mu^{(1,1)} F(N, p, 0,1,1,1)+2 \mu^{(0,1)} \mu^{(2,0)} F(N, p, 0,2,1,0) \\
& +4 \mu^{(1,0)} \mu^{(1,1)} F(N, p, 1,1,0,1)+2^{\frac{3}{2}} \mu^{(1,0)} \mu^{(2,0)} F(N, p, 1,2,0,0) \\
& +2^{\frac{3}{2}} \mu^{(1,1)} \mu^{(2,0)} F(N, p, 1,2,1,0)
\end{aligned}
$$

where we have used the obvious symmetry property: $F\left(N, p, q_{1}, q_{2}, \alpha_{1}, \alpha_{2}\right)=F\left(N, p, q_{2}, q_{1}, \alpha_{2}, \alpha_{1}\right)$. Substituting Eqs.(4.20) and using the fact that $F\left(N, p, q_{1}, q_{2}, \alpha_{1}, \alpha_{2}\right)=0$ for odd values of $p+$ $q_{1}+q_{2}$ one gets:

$$
\begin{aligned}
\tilde{\chi}_{0,2}= & F(0,2,0,0,0,0)+\frac{1}{4} A^{2} F(0,2,0,0,1,1)+4 B^{2} F(0,2,1,1,0,0) \\
& +\frac{16}{25} B^{2} F(0,2,1,1,1,1)+\frac{9}{4} A^{2} F(0,2,2,2,0,0)-A F(0,2,0,0,0,1) \\
& +3 A F(0,2,0,2,0,0)-\frac{3}{2} A^{2} F(0,2,0,2,1,0)-\frac{16}{5} B^{2} F(0,2,1,1,0,1) \\
= & (1+\alpha) \sqrt{2} \pi^{\frac{7}{2}}\left\{\frac{2}{3}(\alpha-1)+\frac{A}{5}(\alpha-5)+\frac{B^{2}}{250}(25 \alpha-49)-\frac{A^{2}}{280}(3 \alpha+37)\right\}
\end{aligned}
$$

Similarly:

$$
\tilde{\chi}_{1,0}=-\epsilon \sqrt{2} \pi^{\frac{7}{2}}\left(2+\frac{3 A^{2}}{40}+\frac{3 B^{2}}{40}\right)
$$


and:

$$
\tilde{\chi}_{1,1}=\frac{\sqrt{2} \pi^{\frac{7}{2}}}{15}(1+\alpha) B\left\{(31 \alpha-49)-\frac{A}{10}(17 \alpha+5)\right\}
$$

\subsubsection{Solution of the system 4.23}

The system Eqs.(4.23) is now completely specified and can be solved numerically. Upon substituting Eqs.(4.25,4.26,4.27) into Eqs.(4.23) one obtains:

$$
\begin{aligned}
& (1+A) \frac{d \log \widetilde{n}}{d \xi}+(1+A) \frac{d \log \widetilde{T}}{d \xi}+\frac{d A}{d \xi}=-\frac{K n}{\widetilde{n} \widetilde{T}} \\
& B \frac{d \log \widetilde{n}}{d \xi}+\frac{3 B}{2} \frac{d \log \widetilde{T}}{d \xi}+\frac{d B}{d \xi}=-\frac{\epsilon}{\sqrt{2 \pi}}\left(2+\frac{3 A^{2}}{40}+\frac{3 B^{2}}{40}\right) \\
& (5+7 A) \frac{d \log \widetilde{n}}{d \xi}+2(5+7 A) \frac{d \log \widetilde{T}}{d \xi}+7 \frac{d A}{d \xi}= \\
& \frac{8(1+\alpha)}{15 \sqrt{2}} B\left\{(31 \alpha-49)-\frac{A}{10}(17 \alpha+5)\right\}-\frac{K n}{\widetilde{n} \widetilde{T}}(5+2 A) \\
& \frac{8}{3}(1-\alpha)-(5-\alpha) A-\frac{B^{2}}{40}\left(\frac{151}{5}-11 \alpha\right)-\frac{A^{2}}{140}(61+39 \alpha)=0
\end{aligned}
$$

Differentiating the constraint yields the differential system:

$$
\begin{aligned}
& (1+A) \frac{d \log \widetilde{n}}{d \xi}+(1+A) \frac{d \log \widetilde{T}}{d \xi}+\frac{d A}{d \xi}=-\frac{K n}{\widetilde{n} \widetilde{T}} \\
& B \frac{d \log \widetilde{n}}{d \xi}+\frac{3 B}{2} \frac{d \log \widetilde{T}}{d \xi}+\frac{d B}{d \xi}=-\frac{\epsilon}{\sqrt{2 \pi}}\left(2+\frac{3 A^{2}}{40}+\frac{3 B^{2}}{40}\right) \\
& (5+7 A) \frac{d \log \widetilde{n}}{d \xi}+2(5+7 A) \frac{d \log \widetilde{T}}{d \xi}+7 \frac{d A}{d \xi}= \\
& \frac{8(1+\alpha)}{15 \sqrt{2}} B\left\{(31 \alpha-49)-\frac{A}{10}(17 \alpha+5)\right\}-\frac{K n}{\widetilde{n} \widetilde{T}}(5+2 A) \\
& \left(\frac{A}{70}(61+39 \alpha)+5-\alpha\right) \frac{d A}{d \xi}+\frac{B}{20}\left(\frac{151}{5}-11 \alpha\right) \frac{d B}{d \xi}=0
\end{aligned}
$$


which should be solved with boundary conditions $\widetilde{n}(0)=\widetilde{T}(0)=1$ and $A(0), B(0)$. The four constants $A(0), B(0), n(0)$ and $T(0)$ are determined by the four equations:

$$
\begin{aligned}
m \frac{n(0)}{2}\left(\frac{2 T(0)}{m}\right)^{\frac{3}{2}} B(0) & =Q_{z}(0) \\
n(0) T(0)(1+A(0)) & =g N_{T} \\
n(0) T(0) & =\frac{m g}{\pi \sigma^{2} K n} \\
\frac{8}{3}(1-\alpha)-(5-\alpha) A(0)-\frac{B^{2}(0)}{40}\left(\frac{151}{5}-11 \alpha\right)-\frac{A^{2}(0)}{140}(61+39 \alpha) & =0
\end{aligned}
$$

The following two figures are plots of $\tilde{n}$ and $\tilde{T}$ as a function of $\xi$ for $B(0)=0.10,0.20,0.25$. The plots exhibita a maximum in the density and a minimum in the temperature which then increases with altitude. This results are in qualitative agreement with earlier studies of vertically vibrated granular gases under gravity $[6,25]$.

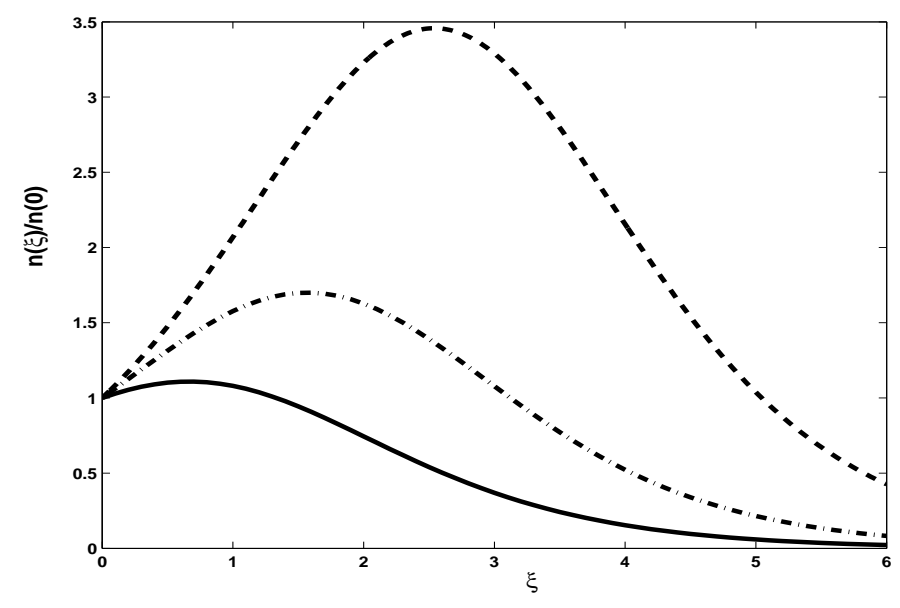

Figure 1: $\widetilde{n}$ as a function of $\xi$ for $K n=0.1, \alpha=0.9$ and $B(0)=0.10$ (solid line, lower curve); $B(0)=0.20$ (dashed line, middle curve); $B(0)=0.25$ (dashed line, upper curve); 


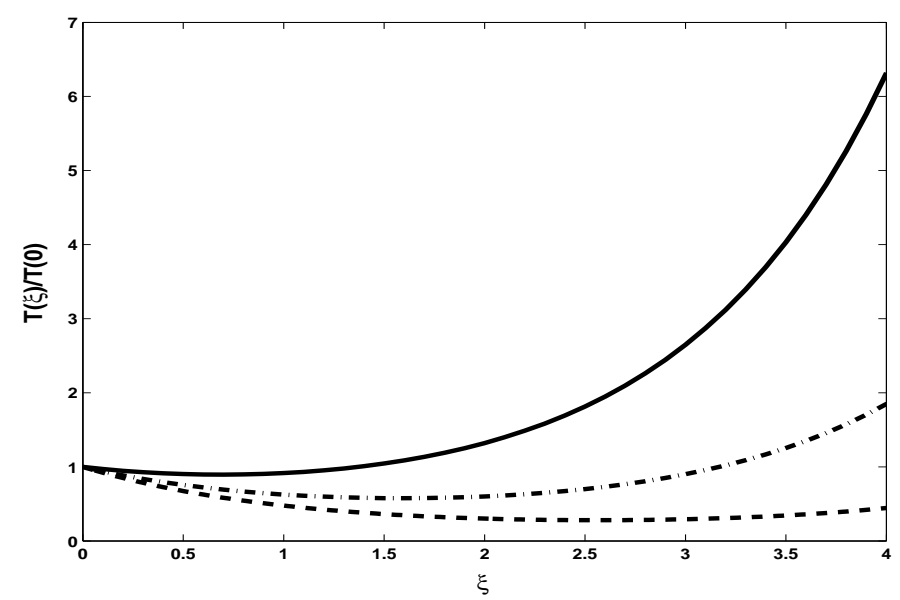

Figure 2: Same as Fig.(1) for $\widetilde{T}$ which exhibits a minimum at $\xi_{\min }: B(0)=0.10, \xi_{\min }=0.7$, $\tilde{T}_{\text {min }}=0.895$ (solid line, upper curve) $B(0)=0.20, \xi_{\text {min }}=0.1, \tilde{T}_{\text {min }}=0.610$ (dashed line, middle curve); $B(0)=0.25, \xi_{\text {min }}=2.52, \tilde{T}_{\min }=0.280$ (dashed line, lower curve);

These results are very close to those obtained by a direct application of Navier-Stokes order in a Chapman-enskog expansion. In the following section we aim at bridging between the Grad 13-moment approach and the Navier-Stokes order.

\subsubsection{Navier-Stokes}

We first rederive the Navier-Stokes system. We refer to [6] for a comprehensive analysis of the problem. The momentum and energy equations are:

$$
\begin{aligned}
\frac{d P_{z z}}{d z} & =-n m g \\
2 \frac{d Q_{z}}{d z} & =-n m \Gamma
\end{aligned}
$$

In [27] the heat flux, the stress tensor and the energy loss term $\Gamma$ have been calculated to second order in the Knudsen number, $K$ (i.e. gradients) and to linear order in $\epsilon$. In the present system one has (the factors of $3^{\frac{3}{2}}$ appear because the definition of temperature in [27] is $\Theta=3 T$ ):

$$
\begin{aligned}
P_{z z} & =n T+O\left(\epsilon^{2}\right) \\
Q_{z} & =-3^{\frac{3}{2}} \widetilde{\kappa} n m \ell \sqrt{T} \frac{d T}{d z}-3^{\frac{3}{2}} \widetilde{\lambda} m \ell T^{\frac{3}{2}} \frac{d n}{d z} \\
\Gamma & =\frac{3^{\frac{3}{2}} \widetilde{\delta}}{\ell} T^{\frac{3}{2}}
\end{aligned}
$$


where

$$
\begin{aligned}
\widetilde{\kappa} & =0.4101+0.1072 \epsilon+O\left(\epsilon^{2}\right) \\
\widetilde{\lambda} & =0.2110 \epsilon+O\left(\epsilon^{2}\right) \\
\widetilde{\delta} & =\sqrt{\frac{16}{27 \pi}} \epsilon-0.1112 \epsilon^{2}+\ldots
\end{aligned}
$$

From Eqs.4.35,4.37,4.38 it is clear that there is a balance between Knudsen order and order in $\epsilon: O\left(K^{2}\right)=O(\epsilon)$. For this reason we shall restrict our calculations to lowest order in $\epsilon$ since we retain linear order in gradients at Navier-stokes order. After changing variable to $\xi$ defined in Eq.(4.11)

$$
\frac{d P_{z z}}{d \xi}=-\ell m n g \equiv-C
$$

where $C=K_{n} n(0) T(0)=K_{n} P_{z z}(0)$ is a constant and

$$
P_{z z}=P_{z z}(0)\left\{1-K_{n} \xi\right\}
$$

Using the identity $\sqrt{T} \frac{d T}{d \xi}=2 T \frac{d \sqrt{T}}{d \xi}$ one has

$$
\begin{aligned}
\frac{Q_{z}}{m 3^{\frac{3}{2}}} & =-2 \widetilde{\kappa} n T \frac{d \sqrt{T}}{d \xi}-\tilde{\lambda} T^{\frac{3}{2}} \frac{d \frac{P_{z z}}{T}}{d \xi} \\
& =-2 P_{z z}(\widetilde{\kappa}-\tilde{\lambda}) \frac{d \sqrt{T}}{d \xi}+\widetilde{\lambda} \sqrt{T} K_{n} P_{z z}(0)
\end{aligned}
$$

Eq.(4.35) reads

$$
\frac{2}{m 3^{\frac{3}{2}}} \frac{d Q_{z}}{d \xi}=-n \widetilde{\delta} T^{\frac{3}{2}}=-\widetilde{\delta} P_{z z} T^{\frac{1}{2}}
$$

finally leading to

$$
\frac{d^{2} \sqrt{T}}{d \xi^{2}}-\frac{2 \widetilde{\kappa}-\widetilde{\lambda}}{2(\widetilde{\kappa}-\widetilde{\lambda})}\left(\frac{K n}{1-K n \xi}\right) \frac{d \sqrt{T}}{d \xi}-\frac{\widetilde{\delta}}{4(\widetilde{\kappa}-\widetilde{\lambda})} \sqrt{T}=0
$$

We consider next the 13-moment Grad expansion. The variables $A$ and $B$ are related to the familiar variables as follows:

$$
\begin{aligned}
A & =\frac{P_{z z}}{n T}-1 \\
B & =\frac{2 Q_{z}}{\rho}\left(\frac{m}{2 T}\right)^{\frac{3}{2}}
\end{aligned}
$$

In Eq.(4.36) we have indicated the dependence on $\epsilon$ to show that $A=O\left(\epsilon^{2}\right)$ and is negligible. Eq. (4.31) shows that $B=O(\sqrt{\epsilon})$ that is $Q_{z}=O(\sqrt{\epsilon})$. The system Eqs. $(4.34,4.29,4.30)$ simplifies 
to:

$$
\begin{aligned}
& \frac{d \log \widetilde{n}}{d \xi}+\frac{d \log \widetilde{T}}{d \xi}=-\frac{K n}{\widetilde{n} \widetilde{T}} \\
& B \frac{d \log \widetilde{n}}{d \xi}+\frac{3 B}{2} \frac{d \log \widetilde{T}}{d \xi}+\frac{d B}{d \xi}=-\sqrt{\frac{2}{\pi} \epsilon} \\
& 5 \frac{d \log \widetilde{n}}{d \xi}+10 \frac{d \log \widetilde{T}}{d \xi}=\frac{8(1+\alpha)}{15 \sqrt{2 \pi}}(31 \alpha-49) B-5 \frac{K n}{\widetilde{n} \widetilde{T}}
\end{aligned}
$$

Eq.(4.40) has solution

$$
\widetilde{n} \widetilde{T}=1-K_{n} \xi
$$

and Eq.(4.42) yields (setting $\alpha=1$ at that order)

$$
B=-\frac{25}{48} \sqrt{2 \pi} \frac{d \log \widetilde{T}}{d \xi}
$$

Substituting into Eq.(4.41)

$$
-\frac{25}{48} \sqrt{2 \pi} \frac{d^{2} \log \widetilde{T}}{d \xi^{2}}-\frac{25}{48} \sqrt{2 \pi} \frac{d \log \widetilde{T}}{d \xi}\left(\frac{d \log \tilde{n}}{d \xi}+\frac{3}{2} \frac{d \log \widetilde{T}}{d \xi}\right)=-\sqrt{\frac{2}{\pi} \epsilon}
$$

or

$$
\frac{d^{2} \log \widetilde{T}}{d \xi^{2}}+\frac{1}{2}\left(\frac{d \log \widetilde{T}}{d \xi}\right)^{2}-\frac{K_{n}}{1-K_{n} \xi} \frac{d \log \widetilde{T}}{d \xi}=\frac{48}{25 \pi} \epsilon
$$

Using $\frac{d \log \widetilde{T}}{d \xi}=\frac{2}{\sqrt{T}} \frac{d \sqrt{T}}{d \xi}$ one finally gets

$$
\frac{d^{2} \sqrt{T}}{d \xi^{2}}-\frac{K_{n}}{1-K_{n} \xi} \frac{d \sqrt{T}}{d \xi}-\frac{24}{25 \pi} \epsilon \sqrt{\widetilde{T}}=0
$$

Eqs. (4.43) and (4.39) are almost identical. The first derivatives are equal at $O(\epsilon)$ while the last term in Eq.(4.39) is equal to $\frac{\widetilde{\delta}}{4(\widetilde{\kappa}-\tilde{\lambda})}=0.2648+O(\epsilon)$ and in Eq. (4.43): $\frac{24}{25 \pi} \epsilon=0.3056 \epsilon$. The difference is only $10 \%$ which is very satisfying. A comprehensive explanation of the connection between Grad's expansion and the Chapman-Enskog expansion should account for the difference. We are not aware of the existence of such an analysis which is surely necessary.

\section{Conclusion}

Our method of generating functions has been applied to the calculation of the source terms in Grad's moment equations. Although we have considered the dilute limit it is possible to include all the terms in Eq.(2.2) (i.e., containing $\Theta$ ). 
To demonstrate the method we have chosen the simple problem of a stationary one-dimensional granular gas under gravity. For clarity we have specialized further to the 13-moment where the calculation is easy to follow and for definiteness we have presented the numerical solution of the system for three typical sets of parameters.

The method presented in this article should find many applications in the field of rarefied gas dynamics since these are "dilute elastic granular gases".

In this article we have tried to revive the standard method of polynomial expansion for solving the Boltzmann equation with the help of modern computer tool of symbolic computation. Despite the mathematical pitfalls of Grad's method of moments we believe it worthwhile to explore the Grad expansion to high moments. Hitherto unknown structures could appear yielding clues for an improved closure mechanism or divergences that could somehow be summed over. Our method provides such a capability.

This work could not have been performed without the support and encouragement of Isaac Goldhirsch. A brilliant physicist, a great teacher and most of all a dear friend. Thank you, Isaac, for all you gave us.

\section{A Supergenerating function}

Define:

$$
I_{1}=\int_{\mathbf{g} \cdot \mathbf{k}>0} d \mathbf{k}(\mathbf{g} \cdot \mathbf{k}) e^{-F} d \mathbf{C}_{1} d \mathbf{C}_{2}
$$

where:

$$
F=b_{1} C_{1}^{2}+b_{2} C_{2}^{2}+b_{3} C_{1}^{\prime 2}+b_{4} C_{2}^{\prime 2}+\frac{x}{2}\left(\mathbf{C}_{1}^{\prime}+\mathbf{C}_{1}\right)^{2}+\frac{y}{2}\left(\mathbf{C}_{1}^{\prime}+\mathbf{C}_{2}\right)^{2}+\frac{z}{2}\left(\mathbf{C}_{1}^{\prime}+\mathbf{C}_{2}^{\prime}\right)^{2}
$$

The kinematical relations are (see Eq.(2.1)):

$$
\begin{aligned}
& \mathbf{C}_{1}^{\prime}=\mathbf{C}_{1}-\frac{1+\alpha}{2}(\mathbf{k} \cdot \mathbf{g}) \mathbf{k} \\
& \mathbf{C}_{2}^{\prime}=\mathbf{C}_{2}+\frac{1+\alpha}{2}(\mathbf{k} \cdot \mathbf{g}) \mathbf{k}
\end{aligned}
$$

Defining the center of mass velocity: $\mathbf{C}_{c m} \equiv \frac{1}{2}\left(\mathbf{C}_{1}+\mathbf{C}_{2}\right)$ one has:

$$
\begin{aligned}
F & =\lambda_{2} \mathbf{C}_{c m}^{2}+\mathbf{q} \cdot \mathbf{C}_{c m}+\left(\frac{\lambda_{2}}{4}-\frac{y+z}{2}\right) g^{2} \\
& +\frac{1+\alpha}{4}\left(-(1-\alpha)\left(b_{3}+b_{4}\right)-\frac{x}{2}(3-\alpha)+\frac{y}{2}(1+\alpha)\right)(\mathbf{k} \cdot \mathbf{g})^{2}
\end{aligned}
$$

where:

$$
\begin{aligned}
\lambda_{2} & =b_{1}+b_{2}+b_{3}+b_{4}+2 x+2 y+2 z \\
\mathbf{q} & =\left(b_{1}-b_{2}+b_{3}-b_{4}+2 x\right) \mathbf{g}-(1+\alpha)\left(b_{3}-b_{4}+x+y\right)(\mathbf{k} \cdot \mathbf{g}) \mathbf{k}
\end{aligned}
$$


Using the well-known Gaussian integral:

$$
\begin{gathered}
\int d^{d} x e^{-\frac{1}{4} \mathbf{x} \mathbf{N}^{-1} \mathbf{x}+\mathbf{q x}}=(4 \pi)^{\frac{d}{2}} \sqrt{|\mathbf{N}|} e^{\mathbf{q}^{\mathbf{T}} \mathbf{N q}} \\
I_{1}=\int_{\mathbf{g} \cdot \mathbf{k}>0} d \mathbf{C}_{c m} d \mathbf{g} d \mathbf{k}(\mathbf{g} \cdot \mathbf{k}) e^{-\lambda_{2} C_{c m}^{2}-\mathbf{q} \cdot \mathbf{C}_{c m}} e^{\frac{1+\alpha}{4}\left((1-\alpha)\left(b_{3}+b_{4}\right)+\frac{x}{2}(3-\alpha)-\frac{y}{2}(1+\alpha)\right)(\mathbf{g} \cdot \mathbf{k})^{2}-\left(\frac{\lambda_{2}}{4}-\frac{y+z}{2}\right) g^{2}}
\end{gathered}
$$

Here, $\mathbf{N}^{-1}=4 \lambda_{2} \mathbf{I}$ where $\mathbf{I}$ is the identity matrix:

$$
\mathbf{q}^{\mathbf{T}} \mathbf{N q}=\frac{1}{4 \lambda_{2}}\left(\left(b_{1}-b_{2}+b_{3}-b_{4}+2 x\right) \mathbf{g}-(1+\alpha)\left(b_{3}-b_{4}+x+y\right)(\mathbf{k} \cdot \mathbf{g}) \mathbf{k}\right)^{2}
$$

Let:

$$
\begin{aligned}
& \lambda_{1}=\frac{\lambda_{2}}{4}-\frac{y+z}{2}-\frac{\left(b_{1}-b_{2}+b_{3}-b_{4}+2 x\right)^{2}}{4 \lambda_{2}} \\
& \lambda_{3}=\frac{1+\alpha}{4}\left(-(1-\alpha)\left(b_{3}+b_{4}+\frac{x+y}{2}\right)+y-x-\frac{1}{\lambda_{2}} A\right)
\end{aligned}
$$

where $A \equiv\left(b_{3}+b_{4}+x+y\right)\left((1+\alpha)\left(b_{3}+b_{4}+x+y\right)-2\left(b_{1}-b_{2}+b_{3}+b_{4}+2 x\right)\right)$ One obtains:

$$
I_{1}=\frac{2 \pi^{\frac{7}{2}}}{\lambda_{2}^{\frac{3}{2}} \lambda_{1}\left(\lambda_{1}+\lambda_{3}\right)}
$$

To obtain the prefactors apply the differential operator to $e^{-F}$ to pull-down the appropriate scalar products. To pull down $C_{1}^{2}$ one replaces the latter quantities by the differential operator $-\frac{\partial}{\partial b_{1}}$. Similarly $C_{2}^{2}$ is replaced by $-\frac{\partial}{\partial b_{2}}, C_{1}^{\prime 2}$ by $-\frac{\partial}{\partial b_{3}}$ and $C_{2}^{\prime 2}$ by $-\frac{\partial}{\partial b_{4}}$. For the scalar products of different velocities proceed as follows. Write:

$$
\begin{aligned}
\mathbf{C}_{1} \cdot \mathbf{C}_{2} & =\frac{1}{2}\left(\mathbf{C}_{1}+\mathbf{C}_{2}\right)^{2}-\frac{1}{2} C_{1}^{2}-\frac{1}{2} C_{2}^{2} \\
& =\frac{1}{2}\left(\mathbf{C}_{1}^{\prime}+\mathbf{C}_{2}^{\prime}\right)^{2}-\frac{1}{2} C_{1}^{2}-\frac{1}{2} C_{2}^{2}=-\frac{\partial}{\partial z}+\frac{1}{2} \frac{\partial}{\partial b_{1}}+\frac{1}{2} \frac{\partial}{\partial b_{2}}
\end{aligned}
$$


Similarly:

$$
\begin{aligned}
\mathbf{C}_{1} \cdot \mathbf{C}_{1}^{\prime} & =\frac{1}{2}\left(\mathbf{C}_{1}^{\prime}+\mathbf{C}_{1}\right)^{2}-\frac{1}{2} C_{1}^{\prime 2}-\frac{1}{2} C_{1}^{2}=-\frac{\partial}{\partial x}+\frac{1}{2} \frac{\partial}{\partial b_{3}}+\frac{1}{2} \frac{\partial}{\partial b_{1}} \\
\mathbf{C}_{1} \cdot \mathbf{C}_{2}^{\prime} & =\mathbf{C}_{1} \cdot\left(\mathbf{C}_{1}+\mathbf{C}_{2}-\mathbf{C}_{1}^{\prime}\right)=C_{1}^{2}+\mathbf{C}_{1} \cdot \mathbf{C}_{2}-\mathbf{C}_{1} \cdot \mathbf{C}_{1}^{\prime} \\
& =-\frac{\partial}{\partial b_{1}}+\left(-\frac{\partial}{\partial z}+\frac{1}{2} \frac{\partial}{\partial b_{1}}+\frac{1}{2} \frac{\partial}{\partial b_{2}}\right)-\left(-\frac{\partial}{\partial x}+\frac{1}{2} \frac{\partial}{\partial b_{3}}+\frac{1}{2} \frac{\partial}{\partial b_{1}}\right) \\
& =\frac{\partial}{\partial x}-\frac{\partial}{\partial z}-\frac{\partial}{\partial b_{1}}+\frac{1}{2} \frac{\partial}{\partial b_{2}}-\frac{1}{2} \frac{\partial}{\partial b_{3}} \\
\mathbf{C}_{2} \cdot \mathbf{C}_{1}^{\prime}= & \frac{1}{2}\left(\mathbf{C}_{1}^{\prime}+\mathbf{C}_{2}\right)^{2}-\frac{1}{2} C_{1}^{\prime 2}-\frac{1}{2} C_{2}^{2}=-\frac{\partial}{\partial y}+\frac{1}{2} \frac{\partial}{\partial b_{3}}+\frac{1}{2} \frac{\partial}{\partial b_{2}} \\
\mathbf{C}_{2} \cdot \mathbf{C}_{2}^{\prime}= & \mathbf{C}_{2} \cdot\left(\mathbf{C}_{1}+\mathbf{C}_{2}-\mathbf{C}_{1}^{\prime}\right)=\mathbf{C}_{1} \cdot \mathbf{C}_{2}+C_{2}^{2}-\mathbf{C}_{1}^{\prime} \cdot \mathbf{C}_{2} \\
= & -\frac{\partial}{\partial z}+\frac{1}{2} \frac{\partial}{\partial b_{1}}+\frac{1}{2} \frac{\partial}{\partial b_{2}}-\frac{\partial}{\partial b_{2}}-\left(-\frac{\partial}{\partial y}+\frac{1}{2} \frac{\partial}{\partial b_{3}}+\frac{1}{2} \frac{\partial}{\partial b_{2}}\right) \\
= & -\frac{\partial}{\partial z}+\frac{\partial}{\partial y}+\frac{1}{2} \frac{\partial}{\partial b_{1}}-\frac{\partial}{\partial b_{2}}-\frac{1}{2} \frac{\partial}{\partial b_{3}} \\
\mathbf{C}_{1}^{\prime} \cdot \mathbf{C}_{2}^{\prime}= & \frac{1}{2}\left(\mathbf{C}_{1}^{\prime}+\mathbf{C}_{2}^{\prime}\right)^{2}-\frac{1}{2} C_{1}^{\prime 2}-\frac{1}{2} C_{2}^{\prime 2}=-\frac{\partial}{\partial z}+\frac{1}{2} \frac{\partial}{\partial b_{3}}+\frac{1}{2} \frac{\partial}{\partial b_{4}}
\end{aligned}
$$

\section{B Maxwell Boundary Conditions}

One of the drawbacks of Grad's method of moments is the determination of the boundary conditions for the higher moments. What should one impose on the vertical flux of the stress tensor in the vertical direction? This question is far from trivial [28]. A very popular choice is the Maxwell boundary conditions (MBC) which is a boundary condition on the whole single particle distribution function and indirectly on its moments (e.g., [30]). The model is defined as follows.

Assume the horizontal bottom wall $(z=0)$ reemits, with probability equal to a (accommodation coefficient), every impinging particle as if drawn from a (wall) Maxwellian distribution, $f_{M}^{w}$ :

$$
f_{M}^{w}(C)=n_{w}\left(\frac{m}{2 \pi T_{w}}\right)^{3 / 2} e^{-\frac{m C^{2}}{2 T_{w}}}
$$

With probability $1-a$ the particle has its velocity along the z-axis reversed. The one particle distribution function $f\left(C_{z}, C_{\perp}, z\right)$ depends on the height, $z$, on $C_{z}$ and on the magnitude of the transverse velocity $C_{T}$. The Maxwell Boundary Condition (MBC) reads:

$$
f\left(C_{z}, C_{\perp}, 0\right)=\Theta\left(C_{z}\right)\left(a f_{M}^{w}(C)+(1-a) f\left(-C_{z}, C_{\perp}, 0\right)\right)+\Theta\left(-C_{z}\right) f\left(C_{z}, C_{\perp}, 0\right)
$$


By continuity at the bottom. :

$$
\begin{aligned}
M_{N, p}(z=0) & =\frac{1}{n(0)} \int \Theta\left(C_{z}\right)\left(a f_{M}^{w}(C)+(1-a) f\left(-C_{z}, C_{T}, 0\right)\right) C^{2 N} C_{z}^{p} d \mathbf{C} \\
& +\frac{1}{n(0)} \int \Theta\left(-C_{z}\right) f\left(C_{z}, C_{T}, 0\right) C^{2 N} v_{z}^{p} d \mathbf{C}
\end{aligned}
$$

Hence:

$$
\int \Theta\left(C_{z}\right)\left(a f_{M}^{w}(C)+(1-a) f\left(-C_{z}, C_{T}, 0\right)\right) C^{2 N} C_{z}^{p} d \mathbf{C}=\int \Theta\left(C_{z}\right) f\left(C_{z}, C_{T}, 0\right) C^{2 N} C_{z}^{p} d \mathbf{C}
$$

which upon substituting (B1) and (4.9) reads:

$$
\begin{aligned}
& a n_{w}\left(\frac{2 T_{w}}{m}\right)^{N+\frac{p}{2}} \int \Theta\left(C_{z}\right) e^{-C^{2}} C^{2 N} C_{z}^{p} d \mathbf{C} \\
+ & (1-a) n(0)\left(\frac{2 T(0)}{m}\right)^{N+\frac{p}{2}} \sum_{\alpha=0}^{\infty} \sum_{q=0}^{\infty} \frac{(-\sqrt{2})^{q}}{q !} \mu^{(q, \alpha)} \int \Theta\left(C_{z}\right) e^{-C^{2}} C^{2(N+\alpha)} C_{z}^{q+p} d \mathbf{C} \\
= & n(0)\left(\frac{2 T(0)}{m}\right)^{N+\frac{p}{2}} \sum_{\alpha=0}^{\infty} \sum_{q=0}^{\infty} \frac{(\sqrt{2})^{q}}{q !} \mu^{(q, \alpha)} \int \Theta\left(C_{z}\right) e^{-C^{2}} C^{2(N+\alpha)} C_{z}^{q+p} d \mathbf{C}
\end{aligned}
$$

or:

$$
a A_{N p}=\frac{n(0)}{n_{w}}\left(\frac{T(0)}{T_{w}}\right)^{N+\frac{p}{2}} \sum_{\alpha=0}^{\infty} \sum_{q=0}^{\infty} \frac{2^{\frac{q}{2}}}{q !} \mu^{(q, \alpha)} A_{N+\alpha, q+p}\left(1-(-)^{q}(1-a)\right)
$$

It is easy to see that the MBC satisfy the three conservation constraints (density, moment, temperature) at the bottom.

\section{Acknowledgments}

D.S. gratefully acknowleges support from the German Science Foundation (DFG) via grant SP608, and from the Alexander von Humboldt Foundation via a Alexander von Humboldt post-doctoral fellowship.

\section{References}

[1] A. E. Beylich. Solving the kinetic equation for all Knudsen numbers. Phys. Fluids 12 (2000), 444-465.

[2] G. A. Bird. Molecular gas dynamics and the direct simulation theory of gas flows. Oxford University Press, 1994. 
[3] M. Bisi, G. Spiga, and G. Toscani. Grad's equations and hydrodynamics for weakly inelastic flows. Phys. Fluids 16 (2004), 4235-4247.

[4] A. V. Bobylev. The Chapman-Enskog and Grad methods for solving the Boltzmann equation. Sov. Phys,. dokl 27 (1982), 29-31.

[5] J. J. Brey, J.W. Dufty, C. S. Kim and A. Santos. Hydrodynamics for granular flows at low density. Phys. Rev. E 58 (1997), 4638-4653.

[6] J. J. Brey, W.-J Ruiz-Montero, and F. Moreno. Hydrodynamics of an open vibrated system. Phys. Rev. E 63 (2001), 061305.

[7] N.V. Briliantov and T. Pöschel. Kinetic theory of granular gases. Oxford University Press, Oxford, 2004.

[8] C. S. Campbell. Rapid granular flows. Annu. Rev. Fluid Mech. 22 (1990), 57-92.

[9] C. Cercignani. Theory and application of the Boltzmann equation. Scottish Acad. Press, Edinburgh and London, 1975.

[10] S. Chapman and T. G. Cowling. The mathematical theory of nonuniform gases. Cambridge University Press, Cambridge, 1970.

[11] L. García-Colin, R. M. Velasco, and F. J. Uribe. Inconsistency in the moment's method for solving the Boltzmann equation. J. Non-Equilib. Thermodyn. 29 (2004), 257-277.

[12] V. Garzó and J. W. Dufty. Dense fluid transport for inelastic hard spheres. Phys. Rev. E 59 (1998), 5895-5911.

[13] I. Goldhirsch. Rapid granular flows. Annu. Rev. Fluid Mech. 35 (2003), 267-293.

[14] S. H. Noskowicz, D. Serero, and O. Bar-Lev. Generating functions and kinetic theory: a computer aided method. Application: constitutive relations for granular gases up to moderate densities. in preparation (2011).

[15] A. Goldshtein and M. Shapiro. Mechanics of collisional motion of granular materials, part 1: general hydrodynamic equations. J. Fluid Mech. 282 (1995), 75-114.

[16] H. Grad. On the kinetic theory of rarefied gases. Commun. Pure Appl. Maths 2 (1949), 331407.

[17] I. N. Ivchenko, S. K. Loyalka, and R.V. Thompson. The polynomial expansion method for boundary value problems of transport in rarefied gases. Z. angew. Math. Phys. 49 (1998), 955-966.

[18] J. T. Jenkins and M. W. Richman. Grad's 13-moment system for a dense gas of inelastic spheres. Arch. Rational. Mech. Anal. 28 (2001), 355-377. 
[19] M. N. Kogan. Rarefied gas dynamics. Plenum, New York, 1969.

[20] C. D. Levermore and W.J. Morokoff. The gaussian moment closure for gas dynamics. SIAM J.App. Math. 59 (1998), 72-96.

[21] D. Mintzer. Generalized orthogonal polynomial solutions of the Boltzmann equation. Phys. Fluids 8 (1965), 1076-1090.

[22] R. Nagai, H. Honma, K. Maeno, and A. Sakurai. Shock wave solution of the Boltzmann kinetic equation in a 13-moment approximation. Shock Waves 13 (2003), 213-220.

[23] S. H. Noskowicz, O. Bar-Lev, D. Serero, and I. Goldhirsch. Computer-aided kinetic theory and granular gases. Europhys. Lett. 79 (2007), 60001.

[24] Y. G. Ohr. Improvement of the grad 13 moment method for strong shock waves. Phys. Fluids 13 (2001), 2105-2114.

[25] R. Ramirez, D. Risso, R. Soto, and P. Cordero. Hydrodynamic theory for granular gases. Phys. Rev. E 62 (2000), 2521-2530.

[26] P. Rosenau, Extending hydrodynamics via the regularization of the Chapman-Enskog expansion. Phys. Rev. A 40 (1989), 7193-7196.

[27] N. Sela and I. Goldhirsch. Hydrodynamic equations for rapid flows of smooth inelastic spheres, to Burnett order. J. Fluid Mech. 361 (1998), 41-74.

[28] R. Soto. Granular systems on a vibrating wall: the kinetic boundary condition. Phys. Rev. E 69 (2004), 61305-61310.

[29] H. Struchtrup and M. Torrilhon. Regularization of Grad's 13 momemt equations: derivation and linear analysis. Phys. Fluids 15 (2003), 2668-2680.

[30] T. Thatcher, Y. Zheng, and H. Struchtrup. Boundary conditions for Grad's 13 moment equations. Progress in Computational Fuid Dynamics 8 (2008), 69-83. 